Check for updates

Cite this: RSC Adv., 2017, 7, 34461

\title{
Mechanical reinforcement of a cellulose aerogel with nanocrystalline cellulose as reinforcer
}

\author{
Tingting Zheng, Ang Li, Zhanying Li, Wenbin Hu, Lin Shao, Lingbin Lu, (D) * Yang Cao \\ and Yongjun Chen
}

As an environmentally friendly aerogel with outstanding performances, the improvement of mechanical properties is urgently required for cellulose aerogel development. In this study, a cellulose aerogel with good mechanical properties was successfully fabricated using nanocrystalline cellulose (NCC) as reinforcer. Characterization results demonstrated that NCC was embedded well into the cellulose aerogel. Tests and evaluation showed that the compressive modulus of the NCC reinforced cellulose aerogel was significantly improved, about 6 times higher than that of the pure cellulose aerogel. Furthermore, after modification with trimethylchlorosilane via a cold plasma treatment, the NCC reinforced aerogel became hydrophobic but its oleophilic property remained. This aerogel showed good adsorption capacity, reached saturation rapidly and possessed good reusability. Reinforced mechanical properties with NCC make this cellulose aerogel a very promising material for highly efficient separation and extraction of organic pollutants and oils from water.

Received 2nd May 2017

Accepted 5th July 2017

DOI: $10.1039 / \mathrm{c} 7 \mathrm{ra0} 4904 \mathrm{~h}$

rsc.li/rsc-advances

reinforce the mechanical properties of cellulose aerogel with NCC as a reinforcer.

\section{Materials and methods}

\subsection{Materials}

Cellulose (cotton linter, $M_{\eta}=1.01 \times 10^{5}$ ), ${ }^{19}$ provided by Hubei Chemical Fiber Co., Ltd, was used without further purification. The $\alpha$-cellulose content was more than $95 \%$. Urea $\left(\left(\mathrm{NH}_{2}\right)_{2} \mathrm{CO}\right)$ and trimethylchlorosilane (TMCS) were purchased from Guanghua Chemical Factory Co., Ltd and Guangdong Aladdin Industrial Corporation, respectively. Sodium hydroxide $(\mathrm{NaOH})$ and sulfuric acid (98 wt\%) were purchased from Guangzhou Chemical Reagents Factory and Luoyang Chemical Factory, respectively. All chemicals and solvents were analytic grade and used as received without further purification unless otherwise stated. Deionized water was used for all experiments unless otherwise mentioned.

\subsection{Preparation of nanocrystalline cellulose}

Nanocrystalline cellulose was prepared by the sulfuric acid hydrolysis method. Cellulose was added to sulfuric acid (64 $\mathrm{wt} \%)$ solution at a ratio of $2: 40(\mathrm{~g}: \mathrm{ml})$. The mixed solution was sonicated at room temperature for $10 \mathrm{~min}$ before hydrolysis reaction. The hydrolysis reaction was conducted at $55{ }^{\circ} \mathrm{C}$ under a constant mechanical stirring for $50 \mathrm{~min}$, and terminated by water quench. The hydrolyzed cellulose was washed twice by centrifugation (5000 rpm, $10 \mathrm{~min}$ and $15{ }^{\circ} \mathrm{C}$ ) to remove excess sulfuric acid. Finally, the NCC suspension was dialyzed against deionized water until a constant $\mathrm{pH}$ was reached. The resulting suspension was stored at $5{ }^{\circ} \mathrm{C}$.
Key Laboratory of Advanced Materials of Tropical Island Resources (Hainan University), Ministry of Education, State Key Laboratory of Marine Resource Utilization in South China Sea, College of Materials and Chemical Engineering, Hainan University, Haikou, China, 570228. E-mail: lulingbin@126.com; Tel: +86 089866279121 


\subsection{Preparation of NCC reinforced cellulose aerogel}

Cellulose aerogel was prepared by a facile method based on our prior study. ${ }^{20}$ Both cellulose and the $\mathrm{NaOH} /$ urea aqueous solution were precooled at $-18{ }^{\circ} \mathrm{C}$, and then mixed with constant stirring at $4{ }^{\circ} \mathrm{C}$ for $10 \mathrm{~min}$. The weight percentages of cellulose, $\mathrm{NaOH}$ and urea in the final mixture were 3.0-5.0 wt\%, $7.0 \mathrm{wt} \%$ and $12.0 \mathrm{wt} \%$, respectively. The same weight of NCC suspension ( $0 \mathrm{wt} \%$ to $3.0 \mathrm{wt} \%$ ) was added into the mixed solution with constant stirring for $5 \mathrm{~min}$. The weight percentages of cellulose (3.0-5.0 wt\%) and NCC (0-3.0 wt\%) meant concentrations of pre-mixture, respectively. Then the solution was injected into a mould and sonicated to eliminate bubbles. Next, a $17 \mathrm{wt} \%$ sulfuric acid solution, as coagulator, was dropped into the cellulose solution slowly. Cellulose gel was obtained after resting 6 h. Subsequently, the gel was washed with deionized water repeatedly, and then froze at $-80^{\circ} \mathrm{C}$. Finally, the aerogel was obtained by freeze-drying for $48 \mathrm{~h}$ under vacuum.

\subsection{Estimation of density and porosity}

The apparent density $\left(\rho_{\mathrm{A}}\right)$ was expressed in the following formula: $\rho_{\mathrm{A}}\left(\mathrm{g} \mathrm{cm}^{-3}\right)=m / V$ where, $m$ and $V$ were weight and volume of aerogel, respectively. The porosity $(P)$ was expressed in the following formula: $P(\%)=1-\rho_{\mathrm{A}} / \rho_{\mathrm{s}}$ where, $\rho_{\mathrm{s}}$ represented the skeletal density of cellulose. $\rho_{\text {s-cellulose }}=1.528 \mathrm{~g} \mathrm{~cm}^{-3}$.

\subsection{Analysis of structure and morphology}

NCC was observed under a JEM 2100 transmission electron microscope (TEM, JOEL Ltd., Japan). Firstly, sample for TEM was prepared by deposition of diluted NCC suspension on a carbon-coated grid (aqueous dispersions). Then the grid was negatively stained with $1.0 \mathrm{wt} \%$ phosphotungstic acid solution and dried under an infrared heating lamp. The voltage of this process was $200 \mathrm{kV}$. The length and width of around $100 \mathrm{NCC}$ was measured by Nano Measurer 1.2.

Surface morphology of aerogel was investigated using a scanning electron microscopy (SEM, S-4800N, Hitachi, Japan), and operated $10.0 \mathrm{kV}$ after coated with $\mathrm{Au}$ to make the sample conductive.

\subsection{Evaluation of mechanical properties}

The mechanical properties of the reinforced aerogel and pure cellulose aerogel were evaluated by compression deformation test. The aerogels were molded as cube $(30 \mathrm{~mm} \times 30 \mathrm{~mm} \times$ $20 \mathrm{~mm}$ ) and dried at $60{ }^{\circ} \mathrm{C}$ for $24 \mathrm{~h}$. Compressive test was performed with a microcomputer controlled electronic universal testing machine (Jinan HengXu Testing Machine Technology Co., Ltd). The sample was placed between testing plates (a load cell of $509.9 \mathrm{~N}$ was used) and compressed to $85-90 \%$ of its original thickness with a speed of $0.3 \mathrm{~mm} \mathrm{~min}^{-1}$. Each sample was measured in triplicate and the data are presented as means \pm standard deviation.

\subsection{Cold plasma hydrophobic modification}

The NCC reinforced cellulose aerogel was modified by a HD-1A cold plasma equipment provided by Chinese Academy of sciences. TMCS was used as the cold plasma, and the modification process was based on the prior study in our group. ${ }^{\mathbf{2 0}}$ Briefly, dry aerogels were placed in the cold plasma chamber and treated under a glow discharge system. The discharge system started when vacuum degree reached a constant value (ca. $200 \pm 50 \mathrm{~Pa}$ in these experiments). Finally, the hydrophobic aerogel was obtained under a specified power $(150 \mathrm{~W})$ and time (3 $\mathrm{min})$.

\subsection{Water contact angle test}

Characterization of hydrophobicity (drop method, $5 \mu \mathrm{l}$ ) was conducted by DropMeterTM Experience A-300 (AST. Ltd, America) equipped with USB2.0 CMOS industrial digital camera (the maximum resolution $1280 \times 1024$ with the speed of $25 \mathrm{fps}$ ). The contact angle of sample was measured and analyzed by DropMeterTM software automatically.

\subsection{The liquid adsorption properties and reusability of aerogel}

The aerogel sample approximately $20 \mathrm{mg}$ was immersed in $50 \mathrm{ml}$ different liquids (such as tap water, peanut oil, ethanol, etc.) for $1 \mathrm{~h}$. The liquid adsorption capacity $\left(C_{\mathrm{m}}\right)$ was indicated as follows: $C_{\mathrm{m}}=\left(m_{1}-m_{0}\right) / m_{0}\left(\mathrm{~g} \mathrm{~g}^{-1}\right)$ where $m_{0}$ and $m_{1}$ were the weight of sample before and after adsorption, respectively. The reusability of aerogel was indicated by repeating the measurement of adsorption capacity for ethanol and pump oil. The liquid adsorption capacity $\left(C_{\mathrm{m}}\right)$ was indicated as follows: $C_{\mathrm{m}}=\left(m_{\mathrm{q}}-m_{\mathrm{n}}\right) / m_{\mathrm{n}}\left(\mathrm{g} \mathrm{g}^{-1}\right)$, where $m_{\mathrm{n}}$ and $m_{\mathrm{q}}$ were the weight of sample before and after adsorption, respectively. After dealt with ethanol via elution to remove the pump oil and dried at $50{ }^{\circ} \mathrm{C}$, the aerogel sample for pump oil was reused.

\section{Results and discussion}

\subsection{Density, porosity and morphology of aerogel}

The bulk density and porosity of aerogel were depicted in Fig. 1. With the increase of NCC concentration, the bulk density of aerogel increased from $0.027 \mathrm{~g} \mathrm{~cm}^{-3}$ to $0.041 \mathrm{~g} \mathrm{~cm}^{-3}$, while the porosity decreased from $98.2 \%$ to $97.3 \%$ continuously.

From TEM image of NCC in Fig. 2a, the individual NCC exhibited a rod-like structure. The width of NCC ranged from 4 to $10 \mathrm{~nm}$, and the length ranged from 50 to $180 \mathrm{~nm}$. The cellulose fibers formed a large space frame structure both in the pure cellulose (Fig. 2b) and NCC reinforced cellulose aerogel (Fig. 2c). In Fig. 2c, NCC was embedded into cellulose aerogel and acted as filler in the void of the structure. Meanwhile, an open porous multidimensional network structure was formed for the NCC reinforced aerogel (Fig. 2d). Thus, it made the NCC reinforced cellulose aerogel more robust and denser than the pure cellulose aerogel, and the bulk density went up at the same time.

\subsection{Mechanical properties of aerogel}

In the present work, NCC was added in cellulose aerogel to enhance its mechanical properties. As shown in Fig. $3 g$, three regions were exhibited: (I) a linear elastic region at a very low 


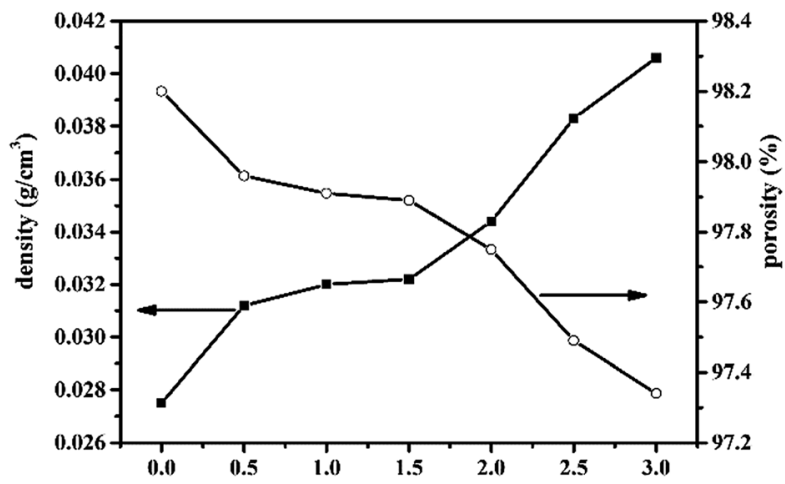

Fig. 1 The effect of NCC suspension concentration on bulk density and porosity of aerogel (cellulose concentration $=3 \mathrm{wt} \%$ ).

strain $(<5 \%)$, followed by (II) a long plastic deformation region with pore walls bending (the transition from linear to nonlinear stress-strain behaviour was gradual, 5-55\%), and (III) densification of the porous structure, which were typical stressstrain curves of porous materials. ${ }^{21}$ Similar compression behaviours of porous bodies were published by several literatures. $^{22,23}$ It was worthy to say that the aerogel showed very high deformability, up to $85-90 \%$ strain without any break (Fig. 3a-c). Meanwhile, the aerogel had not cracked even though folded to $180^{\circ}$ (Fig. 3e and f). The property was better than that of those aerogels made from silica (easy to break). ${ }^{24,25}$

The compressive modulus was determined from the slope of the linear region of the curves Fig. 3g. Pekala et al. ${ }^{26}$ called this value "compressive modulus" instead of Young' modulus. The latter connotes a property of a linear-elastic material which other aerogels are not. According to Karadagli et al. ${ }^{22}$ the yield strength is as the cross point of the deformation curve with a line drawn parallel to the linear-elastic line at $0.2 \%$ strain, as shown in Table 1.

The compressive modulus of pure cellulose aerogel increased from $69.2 \mathrm{kPa}$ to $207.1 \mathrm{kPa}$ with increasing pure cellulose content from 2 to $2.5 \mathrm{wt} \%$ (Table 1). This result indicated that mechanical properties of cellulose aerogel were

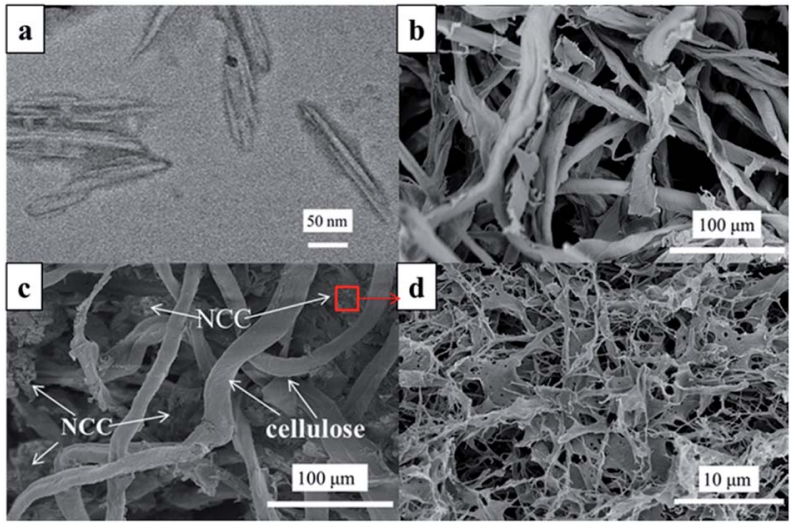

Fig. 2 (a) TEM image of NCC; (b) SEM image of pure cellulose aerogel; (c and d) SEM images of the NCC reinforced cellulose aerogel (cellulose : NCC $=3: 0.5$ ). correlated with the solid content. Furthermore, the NCC reinforced cellulose aerogel also showed this phenomenon. But the compressive modulus and yield strength of the reinforced aerogel were far higher than those of the pure cellulose aerogel. The compressive modulus of the reinforced aerogel (sample D, $402.8 \mathrm{kPa}$ ) was nearly 6 times higher than that of the pure cellulose aerogel (sample A, $69.2 \mathrm{kPa}$ ) with the same total solid content ( $2 \mathrm{wt} \%)$. Even at a lower total solid content level, the compressive modulus of the reinforced aerogel was still higher than that of the pure cellulose aerogel with a higher total solid content. It indicated that the addition of NCC in cellulose aerogel largely improved its mechanical properties. This result was consistent with the result of previous SEM analysis. NCC acted as filler in the void of aerogel structure and formed a rigid structure, which provided a strong support for the whole cellulose aerogel. Compared to other reinforcers, such as silica, the compressive modulus of the NCC reinforced cellulose aerogel (213.8-489.1 kPa) was 2-4 times higher than that of the silica-cellulose aerogel (86-169 kPa)10. Therefore, NCC was a more effective and potential reinforcer for cellulose aerogel.

\subsection{Modification and liquid adsorption properties of aerogel}

Nature cellulose is rich in hydroxyl and methylene groups, resulting amphipathy (both hydrophilic and oleophilic properties) for cellulose aerogel. Thus, it is a big difficulty for cellulose

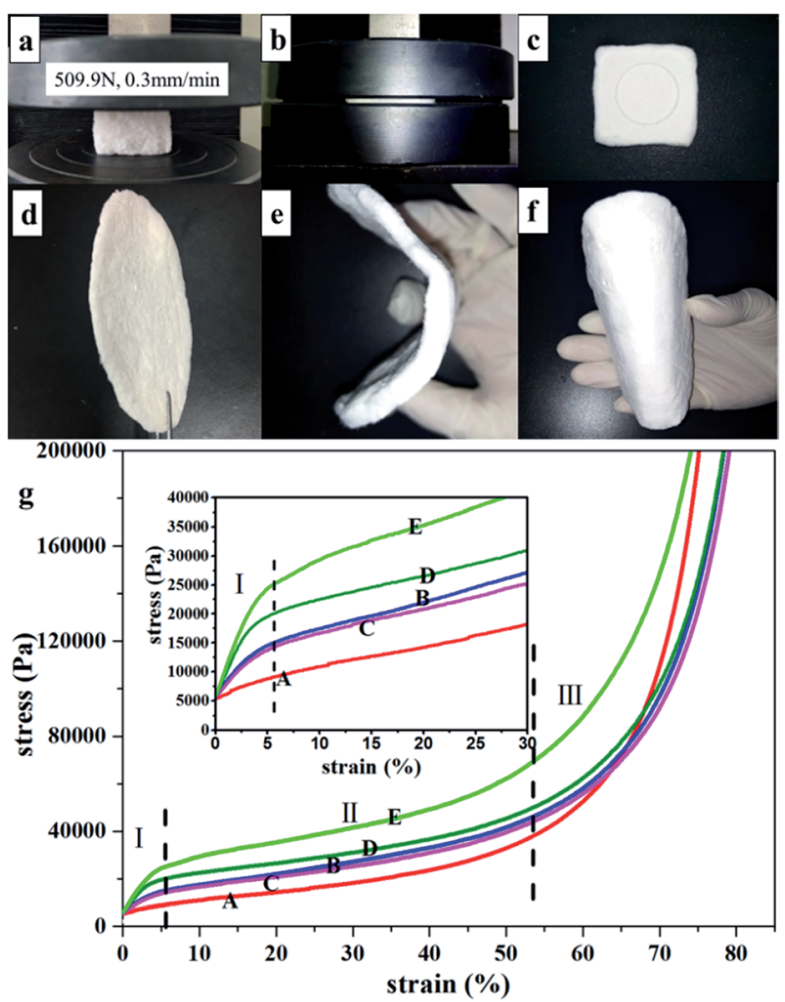

Fig. 3 Photos of aerogel before (a) and after ( $b$ and c) compressed, before ( $d$ ) and after (e and f) folded; ( $g$ ) typical uniaxial compression stress-strain curves for pure cellulose aerogels (A and B) and NCC reinforced cellulose aerogels ( $C, D$ and $E$ ), the linear elastic region was zoomed in and displayed in the inset. 
Table 1 Mechanical properties of aerogels

\begin{tabular}{lllll}
\hline Aerogel sample & Cellulose $:$ NCC $(\mathrm{m} \mathrm{:} \mathrm{m})^{a}$ & Total solid content ${ }^{b}(\mathrm{wt} \%)$ & Compressive modulus $(\mathrm{kPa})^{\text {Yield strength }(\mathrm{kPa})}$ \\
\hline A & $4.0: 0$ & 2.00 & $69.2 \pm 3.10$ & $14.7 \pm 0.40$ \\
B & $5.0: 0$ & 2.50 & $207.1 \pm 8.66$ & $16.6 \pm 0.42$ \\
C & $3.0: 0.5$ & 1.75 & $213.8 \pm 9.17$ & $15.4 \pm 0.36$ \\
D & $3.0: 1.0$ & 2.00 & $402.8 \pm 15.08$ & $19.6 \pm 0.50$ \\
E & $3.0: 1.5$ & 2.25 & $489.1 \pm 18.25$ & $24.8 \pm 0.61$
\end{tabular}

${ }^{a}$ The content ratio of cellulose to NCC in final sample. ${ }^{b}$ The total content of NCC and cellulose in the final hydrogel sample.

aerogel to remove organic contaminants from water. Therefore, to achieve hydrophobic oil-adsorbing material, the reinforced aerogel was modified in this work.

As reported in our previous work, ${ }^{20}$ the aerogel was grafted $-\mathrm{Si}\left(\mathrm{CH}_{3}\right)_{3}$ after modified via the cold plasma technology with TMCS as a plasma. The silylation procedure was showed in Fig. 4. This technology is a facile, effective and rapid method for hydrophobic modification of aerogel.

As shown in Fig. 5a, the unmodified aerogel was amphiphilic. In the Fig. $5 c$, the water contact angle of the modified NCC reinforced cellulose aerogel was $143.8^{\circ}$. So the modified aerogel exhibited hydrophobic feature and remained oleophilic feature (Fig. 5b), which helped to achieve oil-water separation. As shown in Fig. 5d, the adsorption capacity for tap water and peanut oil increased rapidly with the decrease of NCC concentration. It was in accordance with the change of aerogel's density. Generally, low density means high porosity and high adsorption capacity. After hydrophobic modification, there was little difference on adsorption capacities for different adsorbates except for water (Fig. 5e). Therefore, the modification would not influence the adsorption capacity of the aerogel.

In order to investigate the reusability of the modified NCC reinforced cellulose aerogel, ten adsorption-desorption cycles were conducted (as shown in Fig. 5f). It was observed that the adsorption capacity of the modified aerogel did not decrease dramatically after the 10th cycling process. Fig. $5 \mathrm{~g}$ showed up adsorption capacity for pump oil of the modified aerogel at different stages. The adsorption capacity had already reached saturation in a short time. The feature of rapid adsorption will be helpful to save time in practical applications.

The adsorption capacity of the NCC reinforced cellulose aerogel ranged from 10 to $17 \mathrm{~g} \mathrm{~g}^{-1}$, which was similar with other cellulose aerogels at the same cellulose content. ${ }^{20,27,28}$ Meanwhile, the adsorption performance of the NCC reinforced cellulose aerogel was comparable with other kinds of adsorbents, such as nonwoven polypropylene $\left(15 \mathrm{~g} \mathrm{~g} \mathrm{~g}^{-1}\right),{ }^{29}$ PDMSbased material $\left(4-11 \mathrm{~g} \mathrm{~g}^{-1}\right),,^{30}$ monolithic silica $\left(14 \mathrm{~g} \mathrm{~g}^{-1}\right)^{31}$ and PU-based graphene foam $\left(9-27 \mathrm{~g} \mathrm{~g}^{-1}\right) .{ }^{32}$ Compared with<smiles>C[Si](C)(C)O[Si](C)(C)Cl</smiles>

Fig. 4 The silylation of cellulose with TMCS.
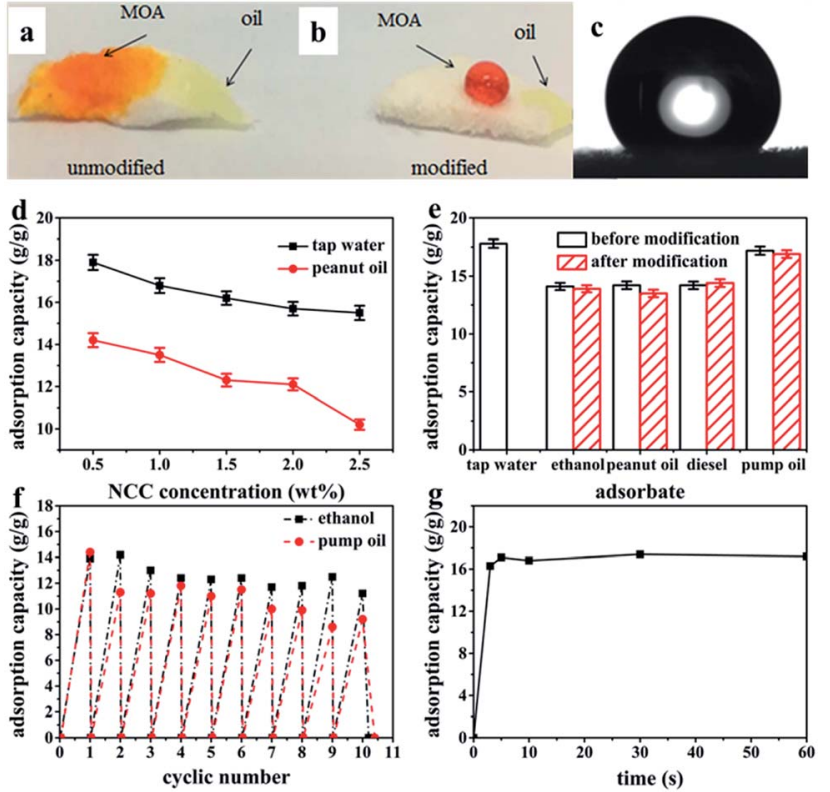

Fig. 5 (a) The unmodified aerogel was amphiphilic; (b) the modified aerogel remained oleophilic but hydrophobic; (c) water contact angle on the cross section of the modified sample $C_{\text {; }}(\mathrm{d})$ adsorption capacities of the NCC reinforced aerogel (before modification) with increasing NCC concentration (cellulose concentration $=3 w t \%$ ) for tap water and peanut oil; (e) adsorption capacities of sample $C$ (before and after modification) for various adsorbates; (f) the cyclic adsorption capacity of sample $C$ for ethanol and sample D for pump oil; (g) adsorption capacity of sample $\mathrm{C}$ for pump oil at different stages. All data were the average value of six replicate samples, the error bar represented the standard deviation.

these adsorbents, the NCC reinforced cellulose aerogel was environmentally friendly and low-cost.

\section{Conclusions}

In this study, mechanical properties of cellulose aerogel were reinforced by NCC successfully. The compressive modulus of the NCC reinforced aerogel was about 2-6 times higher than that of the pure cellulose aerogel. The modified NCC reinforced aerogel exhibited both hydrophobicity and oleophilicity with a water contact angle up to $143.8^{\circ}$. The adsorption capacity of aerogel was up to $17 \mathrm{~g} \mathrm{~g}^{-1}$ with a fast adsorption speed for pump oil, and this aerogel could be reused more than 10 times. As a result, the modified NCC reinforced aerogel was not only with 
better mechanical properties, but also good adsorbability and high reusability. The effective reinforcement of NCC made cellulose aerogel a more promising material for many applications, such as removal of oil-spill pollutants and organic solvents from water.

\section{Conflict of interest}

The authors declare no competing financial interest.

\section{Acknowledgements}

The authors acknowledge financial support from the National Natural Science Foundation of China (No. 51361009) and Key Laboratory of Advanced Materials of Tropical Island Resources (Hainan University) of Ministry of Education (No. AM2017-23).

\section{Notes and references}

1 J. Fricke, J. Non-Cryst. Solids, 1988, 100, 169.

2 N. Yan, Y. Zhou, Y. Zheng, S. Qiao, Q. Yu, Z. Li and H. Lu, RSC Adv., 2015, 5, 97467.

3 S. Yang, L. Chen, L. Mu, B. Hao and P. C. Ma, $R S C A d v ., 2015$, 5,38470 .

4 S. He, Y. Zhang, X. Shi, Y. Bi, X. Luo and L. Zhang, Mater. Lett., 2015, 144, 82.

5 A. Shinko, S. C. Jana and M. A. Meador, $R S C A d v ., 2015,5$, 105329.

6 L. Zhang, J. Wu, X. Zhang, G. Gong, J. Liu and L. Guo, RSC Adv., 2015, 5, 12592.

7 S. S. Kistler, Nature, 1931, 127, 741.

8 J. Kalmár, M. Kéri, Z. Erdei, I. Bányai, I. Lázár, G. Lente and I. Fábián, RSC Adv., 2015, 5, 107237.

9 H. Schäfer, B. Milow and L. Ratke, RSC Adv., 2013, 3, 15263. 10 J. Feng, D. Le, S. T. Nguyen, V. T. C. Nien, D. Jewell and M. D. Hai, Colloids Surf., A, 2016, 506, 298.

11 F. He, C. Sui, X. He and M. Li, Mater. Lett., 2015, 152, 9.

12 W. P. Flauzino Neto, H. A. Silvério, N. O. Dantas and D. Pasquini, Ind. Crops Prod., 2013, 42, 480.
13 Y. Habibi, L. A. Lucia and O. J. Rojas, Chem. Rev., 2010, 110, 3479.

14 M. J. Cho and B. D. Park, J. Ind. Eng. Chem., 2011, 17, 36.

15 A. Kumar, K. M. Rao, S. E. Kwon, N. L. Yu and S. S. Han, Mater. Lett., 2017, 193, 274.

16 H.-Y. Mi, X. Jing, J. Peng, M. R. Salick, X.-F. Peng and L.-S. Turng, Cellulose, 2014, 21, 2727.

17 M. D. Gawryla, O. V. Den Berg, C. Weder and D. A. Schiraldi, J. Maroc. Chim. Heterocycl., 2009, 19, 2118.

18 A. Kumar, Y. Lee, D. Kim, K. M. Rao, J. Kim, S. Park, A. Haider, D. H. Lee and S. S. Han, Int. J. Biol. Macromol., 2016, 95, 962.

19 W. Brown and R. Wikström, Eur. Polym. J., 1965, 1, 1.

20 R. Lin, A. Li, T. Zheng, L. Lu and Y. Cao, RSC Adv., 2015, 5, 82027.

21 A. Demilecamps, C. Beauger, C. Hildenbrand, A. Rigacci and T. Budtova, Carbohydr. Polym., 2015, 122, 293.

22 I. Karadagli, B. Schulz, M. Schestakow, B. Milow, T. Gries and L. Ratke, J. Supercrit. Fluids, 2015, 106, 105.

23 M. Schestakow, I. Karadagli and L. Ratke, Carbohydr. Polym., 2016, 137, 642.

24 A. C. Pierre and G. M. Pajonk, Chem. Rev., 2002, 102, 4243.

25 H. Maleki, L. Durães and A. Portugal, J. Non-Cryst. Solids, 2014, 385, 55.

26 R. W. Pekala, C. T. Alviso and J. D. Lemay, J. Non-Cryst. Solids, 1990, 125, 67.

27 J. D. Feng, S. T. Nguyen and H. M. Duong, Adv. Mater. Res., 2014, 936, 938.

28 C. Wan and L. Jian, Carbohydr. Polym., 2016, 150, 172.

29 M. Radetic, V. Ilic, D. Radojevic, R. Miladinovic, D. Jocic and P. Jovancic, Chemosphere, 2008, 70, 525.

30 S. J. Choi, T. H. Kwon, H. Im, D. I. Moon, D. J. Baek, M. L. Seol, J. P. Duarte and Y. K. Choi, ACS Appl. Mater. Interfaces, 2011, 3, 4552.

31 T. Sun, H. Fan, Z. Wang and Z. Wu, J. Mater. Sci., 2013, 48, 6713.

32 C. Liu, J. Yang, Y. Tang, L. Yin, H. Tang and C. Li, Colloids Surf., A, 2015, 468, 10. 